\title{
The density, the cosmic microwave background, and the proton-to-electron mass ratio in a cloud at redshift $\mathbf{0 . 9}$
}

\author{
C. Henkel ${ }^{1}$, K. M. Menten ${ }^{1}$, M. T. Murphy ${ }^{2}$, N. Jethava ${ }^{3}$, V. V. Flambaum ${ }^{4}$, J. A. Braatz ${ }^{5}$, S. Muller ${ }^{6,7}$, \\ J. Ott ${ }^{5,8, \star}$, and R. Q. $\mathrm{Mao}^{9}$
}

1 Max-Planck-Institut für Radioastronomie, Auf dem Hügel 69, 53121 Bonn, Germany e-mail: chenkel@mpifr-bonn.mpg.de

2 Centre for Astrophysics and Supercomputing, Swinburne University, PO Box 218, Victoria 3122, Australia

3 National Institute of Standards and Technology, M.S. 817.03, 325 Broadway, 80305 Boulder, USA

4 School of Physics, University of New South Wales, Sydney, N.S.W. 2052, Australia

5 National Radio Astronomy Observatory, 520 Edgemont Road, Charlottesville, VA 22903, USA

6 Academia Sinica Institute of Astronomy and Astrophysics, PO Box 23-141, Taipei, 106, Taiwan

7 Onsala Space Observatory, 439-92 Onsala, Sweden

8 CalTech, 1200 E. California Blvd., Caltech Astronomy, 105-24, Pasadena, CA 91125-2400, USA

9 Purple Mountain Observatory, Chinese Academy of Sciences, 210008 Nanjing, PR China

Received 4 December 2008 / Accepted 26 March 2009

\section{ABSTRACT}

\begin{abstract}
Based on measurements with the Effelsberg 100-m telescope, a multi-line study of molecular species is presented toward the gravitational lens system PKS 1830-211, which is by far the best known target to study dense cool gas in absorption at intermediate redshift. Determining average radial velocities and performing Large Velocity Gradient radiative transfer calculations, the aims of this study are (1) to determine the density of the gas, (2) to constrain the temperature of the cosmic microwave background (CMB), and (3) to evaluate the proton-to-electron mass ratio at redshift $z \sim 0.89$. Analyzing data from six rotational $\mathrm{HC}_{3} \mathrm{~N}$ transitions (this includes the $J=7 \leftarrow 6$ line, which is likely detected for the first time in the interstellar medium) we obtain $n\left(\mathrm{H}_{2}\right) \sim 2600 \mathrm{~cm}^{-3}$ for the gas density of the south-western absorption component, assuming a background source covering factor, which is independent of frequency. With a possibly more realistic frequency dependence proportional to $v^{0.5}$ (the maximal exponent permitted by observational boundary conditions), $n\left(\mathrm{H}_{2}\right) \sim 1700 \mathrm{~cm}^{-3}$. Again toward the south-western source, excitation temperatures of molecular species with optically thin lines and higher rotational constants are, on average, consistent with the expected temperature of the cosmic microwave background, $T_{\mathrm{CMB}}=5.14 \mathrm{~K}$. However, individually, there is a surprisingly large scatter which far surpasses expected uncertainties. A comparison of CS $J=1 \leftarrow 0$ and $4 \leftarrow 3$ optical depths toward the weaker north-western absorption component results in $T_{\text {ex }}=11 \mathrm{~K}$ and a $1-\sigma$ error of $3 \mathrm{~K}$. For the main component, a comparison of velocities determined from ten optically thin $\mathrm{NH}_{3}$ inversion lines with those from five optically thin rotational transitions of $\mathrm{HC}_{3} \mathrm{~N}$, observed at similar frequencies, constrains potential variations of the proton-to-electron mass ratio $\mu$ to $\Delta \mu / \mu<1.4 \times 10^{-6}$ with $3-\sigma$ confidence. Also including optically thin rotational lines from other molecular species, it is emphasized that systematic errors are $\Delta V<1 \mathrm{~km} \mathrm{~s}^{-1}$, corresponding to $\Delta \mu / \mu<1.0 \times 10^{-6}$.
\end{abstract}

Key words. galaxies: abundances - galaxies: ISM - galaxies: individual: PKS 1830-211 - gravitational lensing radio lines: galaxies - elementary particles

\section{Introduction}

In the Galaxy, detailed studies of molecular clouds and complexes can be performed at subparsec resolution with a large number of spectroscopic tools in a large variety of regions. However, even interferometric data from nearby galaxies outside the Local Group only provide typical angular resolutions of a few $10 \mathrm{pc}$ for quasi-thermal line emission, matching the size of entire giant molecular clouds. Studies of quasi-thermal line emission from even more distant targets are hampered by small beam filling factors and low flux densities. To circumvent these problems, one can search for masers which may be strong enough to be seen out to cosmological distances (Impellizzeri et al. 2008). Similarly promising is the study of absorption lines. In this case the "effective beamwidth" is determined by the size of the background continuum source, which may be much

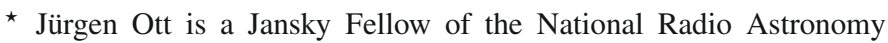
Observatory (NRAO). more compact than the main beam of a single-dish telescope or the synthesized beam of an interferometer system. It is then the strength of the background continuum, which determines the sensitivity of the observations.

In the following we will discuss spectroscopic data obtained toward a particularly prominent radio source, which is observed at an intermediate redshift $(z \sim 0.89),(1)$ to explore the physical properties of a distant molecular cloud, (2) to use these properties for a test of the correlation between $T_{\mathrm{CMB}}$ and redshift, and (3) to search for variations of the proton-to-electron mass ratio as a function of time.

So far, only a few studies exist of individual giant molecular clouds at intermediate redshift (see, Wiklind \& Combes 1994, 1995, 1996a,b; Kanekar et al. 2005). A good knowledge of their physical and chemical conditions is, however, mandatory for an evaluation of systematic differences between clouds at small and large cosmological distances. 
According to the standard model of cosmology, the cosmic microwave background (CMB) should follow a Planck curve of temperature $T_{\mathrm{CMB}}=(2.725 \pm 0.001) \times(1+z)$, with $0 \leq z \leq$ $1088_{-2}^{+1}$ denoting redshift (e.g., Battistelli et al. 2002; Fixsen \& Mather 2002; Spergel et al. 2003). Determining deviations from this relation would be a powerful tool to challenge the standard model of cosmology, highlighting possible mechanisms acting upon the photons of the cosmic microwave background (e.g., Lima et al. 2000). The best experimental method to measure $T_{\mathrm{CMB}}(1+z)$ is a multi-spectral line analysis of ions, atoms or molecules that experience negligible excitation by particle collisions and by radiation from nearby sources, thus being almost exclusively excited by the isotropic radiation of the CMB. A comparison of line temperatures then yields excitation temperatures, which provide firm limits to $T_{\mathrm{CMB}}$.

According to the standard model of particle physics, fundamental constants, at least in their low-energy limits, should be independent of time and location. The fundamental constants of physics and astronomy are well defined locally. Although there have been many detailed studies searching for spatial or temporal variations, none have been convincingly established (e.g., Uzan 2003; García-Berro et al. 2007; Flambaum 2008). However, this "constancy of constants" may not necessarily hold over the largest spatial and temporal scales, which are inaccessible by geological or astronomical studies of nearby targets. Now, advances in observational sensitivity make it possible to measure fundamental constants for sources in the distant Universe, viewed at times billions of years ago and preceding the formation of the solar system (e.g., Bahcall et al. 1967; Varshalovich \& Levshakov 1993; Cowie \& Songaila 1995; Webb et al. 1999).

When searching for variations of physical constants during the last $\sim 10^{10} \mathrm{yr}$, the most frequently studied parameter is the fine-structure constant $\alpha$ (Webb et al. 1999, 2001; Murphy et al. 2003, 2008b; Chand et al. 2004; Levshakov et al. 2006, 2007; Rosenband et al. 2008). Composite parameters consisting of $\alpha$, the proton-to-electron mass ratio $\mu$, and the proton- $g$ factor $g_{\mathrm{p}}$ have also been analyzed (Drinkwater et al. 1998; Carilli et al. 2000; Murphy et al. 2001; Chengalur \& Kanekar 2003; Kanekar et al. 2004, 2005; Tzanavaris et al. 2005, 2007). It may well be possible that the chromodynamic scale varies faster than the corresponding scale of quantum-electrodynamics and that, as a consequence, the proton-to-electron mass ratio $\mu$ may show larger deviations from local values than the fine-structure constant (e.g., Calmet \& Fritzsch 2002; Langacker et al. 2002; Flambaum et al. 2004) though this is highly model dependent (Dent 2008).

To date, studies focusing specifically on potential variations of $\mu$ are rare, yielding $1 \sigma$ confidence levels of order $\Delta \mu / \mu \sim 10^{-5}$ (Levshakov et al. 2002; Ivanchik et al. 2005; Reinhold et al. 2006; King et al. 2008). Different powers of $\mu$ define the scales of electronic, vibrational, and rotational intervals in molecular spectra so that ro-vibronic transitions of the $\mathrm{H}_{2}$ molecule, observed toward distant quasars, could be used.

An alternative and potentially even more precise approach is the analysis of the inversion line spectrum of ammonia $\left(\mathrm{NH}_{3}\right)$. Variations of $\mu$ could be identified as shifts in the relative frequencies of the inversion versus those of the rotational lines. After $\mathrm{NH}_{3}$ had been observed for the first time at significant redshift, in B0218+357 at $z=0.685$ (Henkel et al. 2005), Flambaum \& Kozlov (2007) compared the frequencies of the three detected inversion lines with those of rotational lines of $\mathrm{CO}, \mathrm{HCO}^{+}$, and $\mathrm{HCN}$ (Wiklind \& Combes 1995; Combes \& Wiklind 1995). This resulted in $|\Delta \mu| / \mu=(0.6 \pm 1.9) \times 10^{-6}$. Even more recently, Murphy et al. (2008a) derived from a more thorough analysis of the ammonia spectra and newly observed $\mathrm{HCO}^{+}$and $\mathrm{HCN}$ data $\Delta \mu / \mu=\left(0.74 \pm 0.47_{\text {stat }} \pm 0.76_{\text {sys }}\right) \times 10^{-6}$. As a cautionary note it has to be emphasized, however, that the $\mathrm{NH}_{3}$ inversion and $\mathrm{HCO}^{+}$and $\mathrm{HCN}$ rotational lines have frequencies, which differ by a factor of about ten, while the morphology of the background continuum is frequency dependent (e.g., Jethava et al. 2007). B0218+357 is located at a lookback time of $6.0 \mathrm{Gyr}$, adopting a standard $\Lambda$-cosmology with $H_{0}=73 \mathrm{~km} \mathrm{~s}^{-1} \mathrm{Mpc}^{-1}$, $\Omega_{\mathrm{m}}=0.28$, and $\Omega_{\Lambda}=0.72$ (Spergel et al. 2007).

Among the redshifted sources showing molecular absorption spectra, the gravitational lens PKS 1830-211 stands out and has distinct advantages over $\mathrm{B} 0218+357$ with respect to line strengths and chemical complexity. With $z=0.88582$ for its main absorption feature and a lookback time of $7.0 \mathrm{Gyr}$, it is also the most distant of these sources presently known. With a lensed $z=2.5$ quasar providing an exceptionally bright radio background, a vast number of molecular absorption lines have been detected (Wiklind \& Combes 1996b, 1998; Gerin et al. 1997; Chengalur et al. 1999; Menten et al. 1999, 2008; Muller et al. 2006). A total of $11 \mathrm{NH}_{3}$ inversion lines has been observed toward the source (Henkel et al. 2008; J. Ott, priv. comm.). With the kinetic temperature obtained from the $\mathrm{NH}_{3}$ data, here we provide the complementary estimate of the density of the gas as well as excitation temperatures, which can be compared with the expected temperature of the CMB. To determine $\Delta \mu / \mu$, frequencies of $\mathrm{NH}_{3}$ inversion lines will be compared with newly measured optically thin rotational lines also detected in the $\lambda=0.7-2.5 \mathrm{~cm}$ wavelength range.

\section{Observations}

The observations were carried out with the primary focus $\lambda=$ $1.9,1.3,1.0$, and $0.7 \mathrm{~cm}$ receivers of the 100-m telescope at Effelsberg/Germany ${ }^{1}$ between August 2001 and March 2002. For full width to half power (FWHP) beamwidths, system temperatures, and aperture efficiencies, see Table 1. The backend was an 8192 channel autocorrelator, which was split into eight segments, covering selected frequency ranges within a total bandwidth of $500 \mathrm{MHz}$. Each of the eight segments had a bandwidth of $40 \mathrm{MHz}$ with 512 channels or, at $0.7 \mathrm{~cm}, 80 \mathrm{MHz}$ with 256 channels, yielding channel spacings of $0.7-2.0 \mathrm{~km} \mathrm{~s}^{-1}$. At 1.3 and $0.7 \mathrm{~cm}$, dual channel receivers were used. Observing with these receivers, therefore at least two of the eight segments were centered at the same frequency to maximize sensitivity by observing simultaneously the two orthogonal linear polarizations.

As already mentioned (Sect. 1), PKS 1830-211 is one of the strongest compact continuum sources in the radio sky. Therefore, pointing could be checked toward the source itself and was found to be accurate to about $5^{\prime \prime}$. The continuum was also used to calibrate the spectral lines to obtain line-tocontinuum flux density ratios, which could be determined with high accuracy (see Sect. 4).

\section{Results}

PKS 1830-211 is composed of a background continuum source at redshift $z=2.507$, possibly a blazar, and a lensing face-on spiral galaxy at $z=0.88582$ along its line-of-sight (Wiklind \& Combes 1996b; Lidman et al. 1999; Courbin et al. 2002;

1 The 100-m telescope at Effelsberg is operated by the Max-PlanckInstitut für Radioastronomie (MPIfR) on behalf of the Max-PlanckGesellschaft (MPG). 
Table 1. Observational parameters.

\begin{tabular}{ccccc}
\hline \hline $\begin{array}{c}v \\
(\mathrm{GHz})\end{array}$ & $\begin{array}{c}\lambda \\
(\mathrm{cm})\end{array}$ & $\begin{array}{c}\theta_{b}^{a} \\
\left({ }^{\prime \prime}\right)\end{array}$ & $\begin{array}{c}T_{\text {sys }}^{b} \\
(\mathrm{~K})\end{array}$ & $\eta_{\mathrm{A}}^{c}$ \\
\hline $13.5-18.7$ & 1.9 & $60-45$ & 40 & $0.39-0.32$ \\
$18.0-26.0$ & 1.3 & $45-35$ & 70 & $0.33-0.26$ \\
$27.0-36.7$ & 1.0 & $32-23$ & 75 & 0.30 \\
$41.1-49.7$ & 0.7 & $23-19$ & 150 & $0.36-0.13$ \\
\hline
\end{tabular}

${ }^{a}$ Full Width to Half Power (FWHP) beam width in arcsec. ${ }^{b}$ System temperatures in units of antenna temperature $\left(T_{\mathrm{A}}^{*}\right){ }^{c}$ Aperture efficiency.

Winn et al. 2002; de Rosa et al. 2005). The lens splits the background continuum into three main components, two compact hotspots, a north-western and a south-eastern one separated by $\sim 1^{\prime \prime}$, and an Einstein ring, which is prominent at low $(\ll 10 \mathrm{GHz})$ frequencies. The bulk of the molecular absorption is seen toward the south-western hotspot (e.g., Frye et al. 1997; Swift et al. 2001).

Figures 1-3 show the line profiles measured at Effelsberg with the ordinate displaying absorption in units of the observed continuum flux density. No significant indications for variable lineshapes were found during the time interval of the observations (Sect. 2), which is consistent with the optical depth monitoring of Muller \& Guélin (2008, their Fig. 5). Since about one third of the total continuum emission is absorbed by the foreground molecular cloud at $3 \mathrm{~mm}$ wavelength (e.g., Frye et al. 1997; Wiklind \& Combes 1998; Swift et al. 2001), the upper panel of Fig. 1 may show a CS profile with intermediate $(\tau \sim 1)$ peak optical depth.

Ignoring the blueshifted feature at $V_{\mathrm{LSR}} \sim-135 \mathrm{~km} \mathrm{~s}^{-1}$, the only spectral component known to absorb the north-eastern continuum hotspot (see, e.g., Sect. 4.1.4; and Wiklind \& Combes 1998; Muller et al. 2006), this CS $J=1 \leftarrow 0$ profile may be decomposed into four velocity components. Gaussian fitting reveals a main feature at $V_{\mathrm{LSR}}=(8 \pm 1) \mathrm{km} \mathrm{s}^{-1}$, a slightly weaker redshifted one at $(18 \pm 1) \mathrm{km} \mathrm{s}^{-1}$, a weak narrow blueshifted feature at $(-8 \pm 1) \mathrm{km} \mathrm{s}^{-1}$, and a similarly weak but broad line wing encompassing several tens of $\mathrm{km} \mathrm{s}^{-1}$. The lineshape roughly matches that of the opacity profiles presented by Muller et al. (2006, their Fig. 5), also indicating (as the lineto-continuum flux density ratio) that the line is not fully saturated. We note, however, that the CS intensity ratio between the main $\left(\sim 8 \mathrm{~km} \mathrm{~s}^{-1}\right)$ and the redshifted component $\left(\sim 18 \mathrm{~km} \mathrm{~s}^{-1}\right)$ is slightly smaller than for the $\mathrm{HNC}$ and $\mathrm{H}^{13} \mathrm{CO}^{+} J=2 \leftarrow 1$ transitions; it is much smaller than for the $\mathrm{HC}^{18} \mathrm{O}^{+} J=2 \leftarrow 1$ line, which must be optically thin. Muller et al. (2006) give peak optical depths of $\tau=1.65,0.27$, and 0.15 for these three lines, respectively (their Table 4 ). The possibility that CS $J=1 \leftarrow 0$ is moderately optically thick $(\tau \sim 1)$ highlights the possibility that the continuum source covering factor may be smaller at $26 \mathrm{GHz}$ than at $3 \mathrm{~mm}$ (see Sect. 4.1). Interestingly, the $J=1 \leftarrow 0$ peak line-to-continuum ratios of CS (Fig. 1) and $\mathrm{C}^{34} \mathrm{~S}$ (Fig. 3) differ by a factor of $\sim 10$, consistent with the corresponding ratio derived by Muller et al. (2006, their Table 7) for the $J=4 \leftarrow 3$ transition.

The $\mathrm{HCO}^{+} J=1 \leftarrow 0$ profile (Fig. 1, lower panel), taken at a time when the $J=2 \leftarrow 1$ opacity was particularly high (Muller \& Guélin 2008), looks quite different. Here even the line wings at velocities only showing weak CS absorption are quite pronounced and the central three velocity components seen in CS $J=1 \leftarrow 0$ appear to have merged. Remarkably, the velocity of the $\mathrm{HCO}^{+}$absorption peak lies near $+20 \mathrm{~km} \mathrm{~s}^{-1}$, well offset

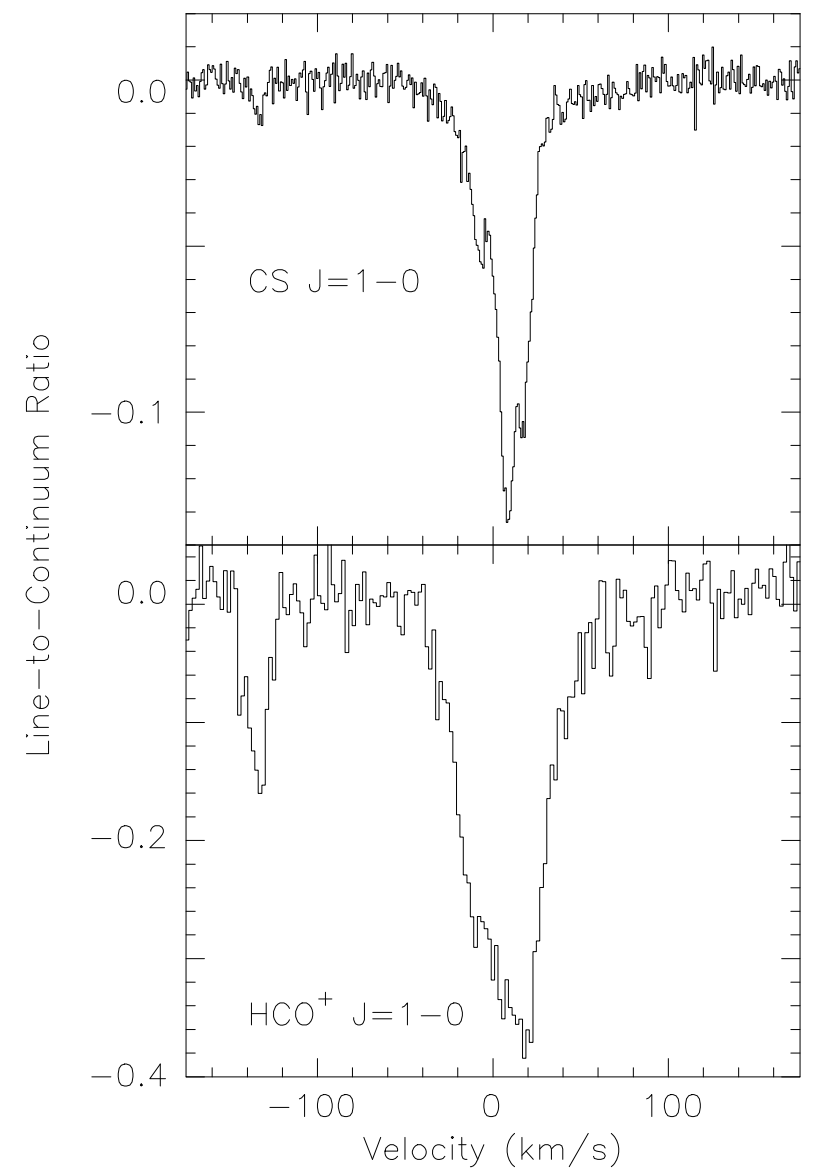

Fig. 1. The CS and $\mathrm{HCO}^{+} J=1 \leftarrow 0$ absorption lines with a Local Standard of Rest (LSR) velocity scale relative to $z=0.88582$, observed toward PKS 1830-211. $V_{\mathrm{LSR}}=V_{\mathrm{HEL}}+12.4 \mathrm{~km} \mathrm{~s}^{-1}$. Channel spacings are 0.9 and $2.0 \mathrm{~km} \mathrm{~s}^{-1}$, respectively. The continuum level accounts for the entire source. Due to a highly frequency dependent noise diode signal at $47.294 \mathrm{GHz}$, the calibration of the $\mathrm{HCO}^{+}$line is uncertain.

from the peak of the CS $J=1 \leftarrow 0$ absorption. The properties of the $\mathrm{HCO}^{+}$line may either be caused by a very large optical depth or by tracing a different volume of gas. Since the $J=2 \leftarrow 1$ lines of the rare $\mathrm{HCO}^{+}$isotopologues show a "normal" optical depth profile (Muller et al. 2006), a high optical depth is the preferred explanation.

Figure 2 shows five detected $\mathrm{HC}_{3} \mathrm{~N}$ profiles. The lines do not absorb more than a few percent of the total continuum and all peak near $8 \mathrm{~km} \mathrm{~s}^{-1}$. From the $J=3 \leftarrow 2$ to the $6 \leftarrow 5$ transition, absolute peak line intensities rise from about $0.5 \%$ to $3.5 \%$ of the background continuum level, only to decrease further up the $J$ ladder. To our knowledge, it is the first time the $\mathrm{HC}_{3} \mathrm{~N} J=7 \leftarrow 6$ line has been detected in the interstellar medium. With its rest frequency of $63.686 \mathrm{GHz}$, it is absorbed by atmospheric $\mathrm{O}_{2}$ in more nearby sources.

Figure 3 shows additional spectral features which also absorb only a tiny fraction of the continuum background. These include transitions of $\mathrm{SiO}$ and rare isotopic species of $\mathrm{CS}, \mathrm{HCN}$, $\mathrm{HCO}^{+}$, and $\mathrm{HNC}$.

\section{Discussion}

\subsection{Excitation analysis}

Before studying molecular excitation in detail, it is important to obtain an estimate of the interplay between the radio continuum 


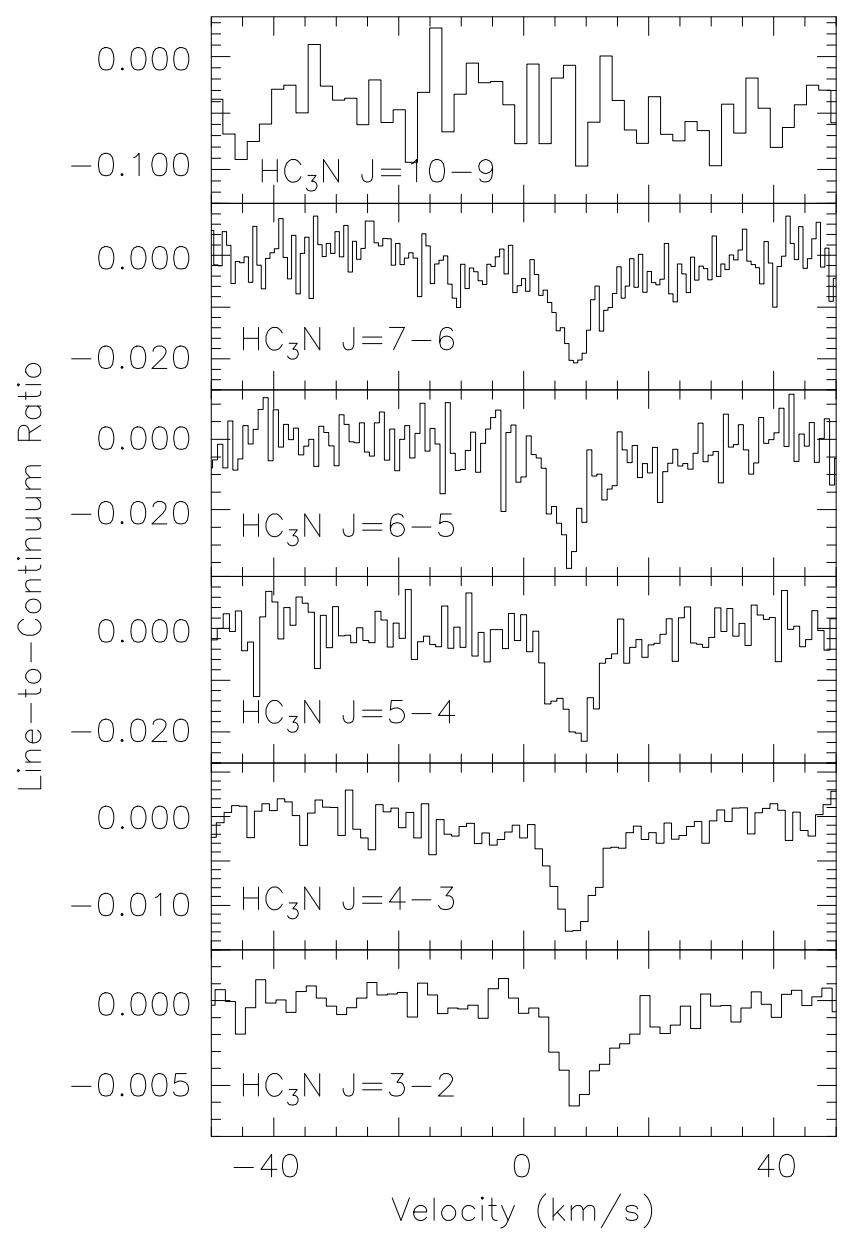

Fig. 2. $\mathrm{HC}_{3} \mathrm{~N}$ absorption lines with a Local Standard of Rest (LSR) velocity scale relative to $z=0.88582$, observed toward PKS 1830-211. Channel spacings are, from top to bottom, 1.9, 0.7, 0.8, 1.0, 1.2, and $1.6 \mathrm{~km} \mathrm{~s}^{-1}$. The continuum level accounts for the entire source.

background and the intervening molecular cloud. PKS 1830-211 is a variable source (e.g., Jin et al. 2003). However, for the limited time interval of our observations we find the depths of the absorption lines and the continuum flux densities to be correlated. This leaves the essential parameter, the line-to-continuum ratio, sufficiently unaffected to combine spectra observed at slighty different times (see also Wiklind \& Combes 1998; Muller et al. 2006; Henkel et al. 2008; Muller \& Guélin 2008). A more intricate problem is the background source coverage factor, which may vary as a function of frequency. Often, the size of the continuum background decreases with frequency (e.g., Lobanov 1998), while the size of the molecular cloud does not vary. If the cloud is located along the line-of-sight to the center of the continuum source, higher frequencies may yield higher source covering factors, while the opposite may hold when the cloud is only absorbing the periphery of the radio background.

At $v \gtrsim 8 \mathrm{GHz}$, the absorbed south-western continuum source of PKS 1830-211 (see Sect. 1) contributes $(38 \pm 2) \%$ to the total radio flux density (e.g., Nair et al. 1993; van Ommen et al. 1995; Carilli et al. 1998; Muller et al. 2006; Menten et al. 2008). According to Frye et al. (1997), the molecular cloud coverage of this continuum component is $\sim 70 \%$. However, Wiklind \& Combes (1998) and Carilli et al. (1998) find values consistent with $100 \%$, which is corroborated by the more recent monitoring results of Muller \& Guélin (2008). This indicates that at a frequency of $94 \mathrm{GHz}$, the covering factor of the total continuum is

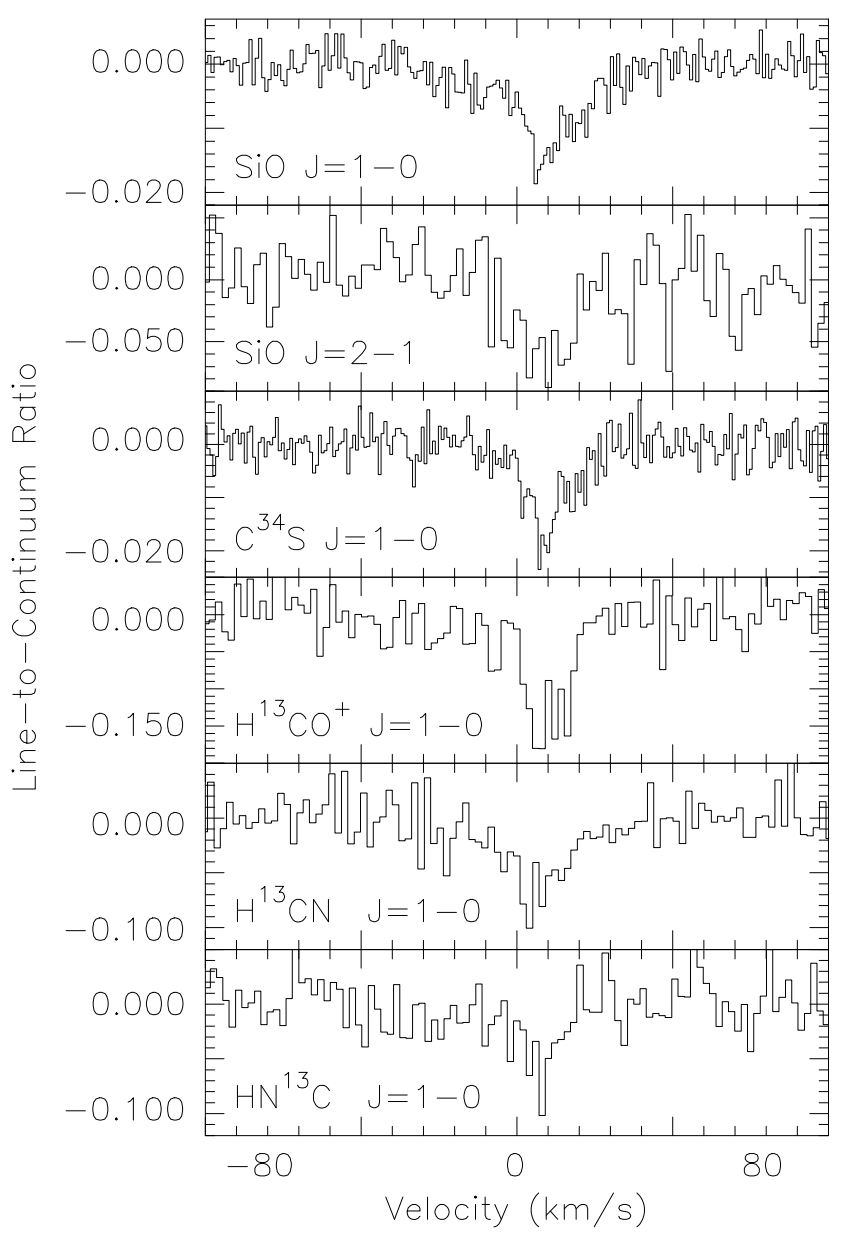

Fig. 3. Additional optically thin absorption lines with a Local Standard of Rest (LSR) velocity scale relative to $z=0.88582$, observed toward PKS 1830-211. Channel spacings are, from top to bottom, 1.0, 2.0, 0.9, $2.0,2.0$, and $2.0 \mathrm{~km} \mathrm{~s}^{-1}$. The adopted continuum level includes the entire source.

indeed $f_{\mathrm{c}}=0.38 \pm 0.02$. With the CS $J=1 \leftarrow 0$ line, redshifted to $26 \mathrm{GHz}$ and absorbing the continuum at a $(13 \pm 1) \%$ level (Sect. 3 and Fig. 1), the variation of $f_{\mathrm{c}}$ between 26 and $94 \mathrm{GHz}$ is then limited to $f_{\mathrm{c}} \propto v^{0.0 \ldots 0.8}$. The upper limit to the exponent would only hold, if the CS $J=1 \leftarrow 0$ line were fully saturated. Because this is certainly not the case (see Sect. 3), the actual maximal exponent must be smaller. For $\tau(\operatorname{CS} J=1 \leftarrow 0)=1$, a reasonable guess implying $f_{\mathrm{c}} \sim 0.2$ at $26 \mathrm{GHz}$, the maximal exponent becomes 0.5 . A negative exponent for $f_{\mathrm{c}}$ as a function of frequency can be excluded because there is no observational evidence for larger source covering factors at lower frequencies (for $\mathrm{OH}$ and HI, see Chengalur et al. 1999; Koopmans \& de Bruyn 2005) and because then the extended, arcsecond sized Einstein ring becomes prominent (e.g., Jauncey et al. 1991).

With five detected and one undetected rotational transition, we can carry out the first multilevel $\mathrm{HC}_{3} \mathrm{~N}$ study of a significantly redshifted source (see Mauersberger et al. 1990, for an analogous study of a nearby starburst galaxy). With its small rotational constant, $\mathrm{HC}_{3} \mathrm{~N}$ is particularly useful to constrain molecular excitation along the line-of-sight to PKS 1830-211. Two $\mathrm{SiO}$ transitions and comparisons of our data with spectra observed at higher frequencies provide additional constraints. The analysis is based on measured "apparent" optical depths,

$\tau_{\text {app }}=-\ln \left(1-\frac{\left|T_{\mathrm{L}}\right|}{T_{\mathrm{C}}}\right)$, 
Table 2. Line widths and optical depths of $\mathrm{HC}_{3} \mathrm{~N}$ transitions (see Sect. 4.1.1) ${ }^{a}$.

\begin{tabular}{cccc}
\hline \hline Line & $F W H P$ & $\tau_{\text {peak }}$ & $\int \tau \mathrm{d} V$ \\
\hline $\mathrm{HC}_{3} \mathrm{~N}=3 \leftarrow 2$ & $10.6 \pm 1.2$ & $0.0057 \pm 0.0008$ & $0.060 \pm 0.005$ \\
$\mathrm{HC}_{3} \mathrm{~N} J=4 \leftarrow 3$ & $9.3 \pm 1.0$ & $0.0131 \pm 0.0018$ & $0.121 \pm 0.010$ \\
$\mathrm{HC}_{3} \mathrm{~N} J=5 \leftarrow 4$ & $8.6 \pm 1.0$ & $0.0211 \pm 0.0031$ & $0.181 \pm 0.017$ \\
$\mathrm{HC}_{3} \mathrm{~N} J=6 \leftarrow 5$ & $9.0 \pm 1.9$ & $0.0280 \pm 0.0069$ & $0.251 \pm 0.034$ \\
$\mathrm{HC}_{3} \mathrm{~N} J=7 \leftarrow 6$ & $12.2 \pm 2.1$ & $0.0173 \pm 0.0035$ & $0.210 \pm 0.024$ \\
$\mathrm{HC}_{3} \mathrm{~N} J=10 \leftarrow 9$ & - & - & $<0.235$ \\
\hline
\end{tabular}

${ }^{a}$ Full Width to Half Power (FWHP) line widths, peak apparent optical depths, and integrated optical depths obtained from one component Gaussian fits. The upper limit to the $\mathrm{HC}_{3} \mathrm{~N} J=10 \leftarrow 9$ integrated opacity is a $3 \sigma$ value.

Table 3. $\mathrm{HC}_{3} \mathrm{~N}$ peak optical depths corrected for incomplete continuum source coverage (see Sect. 4.1.1). $f_{\mathrm{c}}$ is the source covering factor, $v$ denotes the redshifted frequency.

\begin{tabular}{ccc}
\hline \hline Line & $f_{\mathrm{c}}=0.3$ & $\left.f_{\mathrm{c}}=0.2 \times v / 26 \mathrm{GHz}\right)^{0.5}$ \\
\hline $\mathrm{HC}_{3} \mathrm{~N} J=3 \leftarrow 2$ & $0.0239 \pm 0.0046$ & $0.0462 \pm 0.0089$ \\
$\mathrm{HC}_{3} \mathrm{~N} J=4 \leftarrow 3$ & $0.0482 \pm 0.0093$ & $0.0807 \pm 0.0156$ \\
$\mathrm{HC}_{3} \mathrm{~N}=5 \leftarrow 4$ & $0.0721 \pm 0.0106$ & $0.1084 \pm 0.0158$ \\
$\mathrm{HC}_{3} \mathrm{~N} J=6 \leftarrow 5$ & $0.1000 \pm 0.0221$ & $0.1367 \pm 0.0302$ \\
$\mathrm{HC}_{3} \mathrm{~N} J=7 \leftarrow 6$ & $0.0837 \pm 0.0174$ & $0.1059 \pm 0.0219$ \\
\hline
\end{tabular}

with $T_{\mathrm{L}}$ and $T_{\mathrm{C}}$ denoting line and continuum temperature and with $\tau_{\text {app }} \sim\left|T_{\mathrm{L}}\right| / T_{\mathrm{C}}$ being a good approximation in the case of optically thin lines (beware of the typo in the corresponding equation of Henkel et al. 2008).

\subsection{1. $\mathrm{HC}_{3} \mathrm{~N}$}

To reproduce the $\mathrm{HC}_{3} \mathrm{~N}$ data (Fig. 2), we use a Large Velocity Gradient (LVG) model including 22 levels up to $92 \mathrm{~K}$ above the ground state. Collision rates were taken from Green \& Chapman (1978). The bulk of the ammonia $\left(\mathrm{NH}_{3}\right)$ column density arises from gas at $T_{\text {kin }} \sim 80 \mathrm{~K}$ (Henkel et al. 2008). This value was adopted. As already mentioned (Sect. 3), the $\mathrm{HC}_{3} \mathrm{~N}$ lines only absorb a few percent of the total continuum background (Fig. 2, Table 2). This is similar to the $\mathrm{NH}_{3}$ lines also seen at cmwavelengths. Since the $\mathrm{NH}_{3}$ lines are definitely optically thin (the hyperfine satellites are at best barely visible), since $\mathrm{HC}_{3} \mathrm{~N}$ and $\mathrm{NH}_{3}$ frequencies are similar, and since the continuum source covering factor is of order $f_{\mathrm{c}}=0.10-0.38$ (Fig. 1 and Sect. 4.1), we conclude that all detected $\mathrm{HC}_{3} \mathrm{~N}$ lines must also be optically thin.

For the LVG code, a spherical cloud geometry was assumed, but with the lines being optically thin, results do not depend on cloud morphology. We consider the integrated optical depths to be more reliable than the peak optical depths. For the input parameters we therefore only adopted the peak optical depth of the very well fitted $J=5 \leftarrow 4$ line, accounting for a continuum source covering factor $f_{\mathrm{c}}=0.3$ and using the ratios of the integrated optical depths to obtain the peak optical depths of the other lines. This results, ignoring potential uncertainties in the continuum coverage, which are difficult to quantify, in the peak optical depths displayed in the central column of Table 3.

As a first approach we neglect collisional excitation and only vary the temperature of the cosmic microwave background to simulate the observational data with minimized reduced $\chi^{2}$ values, $\chi_{\text {red }}^{2}=\chi^{2} /(N-P)(N=5$ : number of lines, $P=2$ : number
Table 4. $\mathrm{HC}_{3} \mathrm{~N}$ LVG models and resulting reduced $\chi^{2}$ values (see Sect. 4.1.1) ${ }^{a}$.

\begin{tabular}{lrrrr}
\hline \hline Model & $\begin{array}{r}T_{\mathrm{CMB}} \\
(\mathrm{K})\end{array}$ & $\begin{array}{r}n\left(\mathrm{H}_{2}\right) \\
\left(\mathrm{cm}^{-3}\right)\end{array}$ & $f_{\mathrm{c}} \propto v^{0.0}$ & $\chi_{\mathrm{c}}^{2} \propto v^{0.5}$ \\
\hline A1 & 5.14 & 0 & 8.1 & 4.1 \\
$\mathrm{~A} 2$ & 6.00 & 0 & 6.0 & 2.4 \\
$\mathrm{~A} 3$ & 8.00 & 0 & 3.3 & 0.8 \\
$\mathrm{~A} 4$ & 10.00 & 0 & 2.0 & 0.5 \\
$\mathrm{~A} 5$ & 12.00 & 0 & 1.4 & 0.6 \\
$\mathrm{~A} 6$ & 15.00 & 0 & 1.0 & 1.0 \\
$\mathrm{~A} 7$ & 20.00 & 0 & 0.8 & 1.7 \\
$\mathrm{~A} 8$ & 25.00 & 0 & 0.9 & 2.2 \\
A9 & 30.00 & 0 & 1.0 & 2.6 \\
$\mathrm{~A} 10$ & 50.00 & 0 & 1.4 & 3.6 \\
& & & & \\
B1 & 5.14 & 0 & 8.1 & 4.1 \\
B2 & 5.14 & 500 & 5.4 & 2.1 \\
B3 & 5.14 & 1000 & 3.3 & 0.8 \\
B4 & 5.14 & 1500 & 1.7 & 0.3 \\
B5 & 5.14 & 1700 & 1.0 & 0.2 \\
B6 & 5.14 & 2000 & 0.8 & 0.3 \\
B7 & 5.14 & 2500 & 0.4 & 0.7 \\
B8 & 5.14 & 2700 & 0.4 & 1.0 \\
B9 & 5.14 & 3000 & 0.5 & 1.6 \\
B10 & 5.14 & 4000 & 2.1 & 4.0 \\
B11 & 5.14 & 5000 & 4.9 & 7.0 \\
\hline & & & &
\end{tabular}

${ }^{a}$ Assumed kinetic temperature: $T_{\text {kin }}=80 \mathrm{~K}$. For the reduced $\chi^{2}$ values with the frequency independent continuum source coverage factor $f_{\mathrm{c}}=$ 0.3 , see Col. 4. Accounting for the maximal frequency dependence of $f_{\mathrm{c}}, f_{\mathrm{c}}=0.2 \times(v / 26 \mathrm{GHz})^{0.5}$, the corresponding reduced $\chi^{2}$ values are given in the last column. A comparison of the results summarized in the last two columns provides a good measure of the uncertainties involved.

of free parameters; see Jethava et al. 2007). At a redshift of $z=0.88582$, we expect $T_{\mathrm{CMB}}=(2.725 \pm 0.001) \times(1+z)=$ $(5.139 \pm 0.002) \mathrm{K}$. With this value, the minimal $\chi_{\text {red }}^{2}$ is an unacceptable 8.1 (Table 4 ). Modifying $T_{\mathrm{CMB}}$, but still only permitting excitation by the cosmic microwave background, the optimal value is obtained with $\chi_{\text {red }}^{2}=0.8$ at $T_{\mathrm{CMB}} \sim 20 \mathrm{~K}$ (model A7). Such a high $T_{\mathrm{CMB}}$ value is also unacceptable. We therefore have to conclude that our model assumptions are too simple.

As the next step to higher complexity, we therefore also include collisions with $\mathrm{H}_{2}$. Keeping $T_{\mathrm{CMB}}$ fixed at 5.14, we calculate the radiative transfer for $500 \mathrm{~cm}^{-3}<n\left(\mathrm{H}_{2}\right)<5000 \mathrm{~cm}^{-3}$. Already at $n\left(\mathrm{H}_{2}\right)=500 \mathrm{~cm}^{-3}$, the quality of the fit is clearly improved (model B2 of Table 4). The optimum is reached at $n\left(\mathrm{H}_{2}\right) \sim 2600 \mathrm{~cm}^{-3}$ (models B7/B8) with $\chi_{\text {red }}^{2}=0.4$. In this case, calculated and measured optical depths differ by about $15 \%$. Since the opacity ratios do not vary with column density as long as the lines are optically thin, this result is not sensitive to the specific choice of $f_{\mathrm{c}}\left(f_{\mathrm{c}} \propto \tau^{-1} \propto N_{\mathrm{HC}_{3} \mathrm{~N}}^{-1}\right)$. Already at $n\left(\mathrm{H}_{2}\right)=500 \mathrm{~cm}^{-3}$, the $\mathrm{HC}_{3} \mathrm{~N} J=1 \leftarrow 0$ line is inverted. At $n\left(\mathrm{H}_{2}\right)=2000 \mathrm{~cm}^{-3}$, the $2 \leftarrow 1$ line is also inverted and at $n\left(\mathrm{H}_{2}\right)=7000 \mathrm{~cm}^{-3}$, the three ground rotational lines of $\mathrm{HC}_{3} \mathrm{~N}$ are all inverted. Thus, at $n\left(\mathrm{H}_{2}\right) \gtrsim 7000 \mathrm{~cm}^{-3}$, we do not expect to obtain a detectable absorption signal in the $J=3 \leftarrow 2$ transition.

Instead of applying a constant $f_{\mathrm{c}}=0.3$, we can also use the opposite extreme, an $f_{\mathrm{c}}$ value with the strongest frequency dependence permitted by the boundary conditions discussed in Sect. 4.1. For $f_{\mathrm{c}}=0.2 \times(v / 26 \mathrm{GHz})^{0.5}$ the resulting optical 
depths are given in the right column of Table 3 . With $f_{\mathrm{c}}$ decreasing with decreasing frequency, the lower $\mathrm{HC}_{3} \mathrm{~N}$ transitions become more prominent $\left(\tau_{\text {real }} \propto f_{\mathrm{c}}^{-1}\right)$ so that less excitation is required to simulate the calculated optical depths. The right column of Table 4 indicates this change. Excluding collisions, the lowest reduced $\chi^{2}$ is reached at $T_{\mathrm{CMB}}=10 \mathrm{~K}$ instead of the previously obtained $20 \mathrm{~K}$. With $T_{\mathrm{CMB}}=5.14 \mathrm{~K}$ and introducing collisions, we get $n\left(\mathrm{H}_{2}\right) \sim 1700 \mathrm{~cm}^{-3}$ instead of $2600 \mathrm{~cm}^{-3}$. Again, calculated and measured line opacities differ by about $\pm 15 \%$. Overall, the $\chi_{\text {red }}^{2}$ values tend to be smaller than those obtained with $f_{\mathrm{c}}=0.3$. This may indicate that the approach with variable $f_{\mathrm{c}}$ is the better one.

To compare our results with those for the slightly less redshifted source B0218+357 (Henkel et al. 2005, 2008; Jethava et al. 2007), we conclude that in PKS1830-211 kinetic temperature and gas density are much higher, while the source covering factor may be less frequency dependent. However, the situation may not be quite as clear as it appears at first sight. The kinetic temperatures were measured with the same tool, namely the inversion lines of ammonia, thus providing an objective comparison. The gas densities, however, are determined in different ways, analyzing formaldehyde $\left(\mathrm{H}_{2} \mathrm{CO}\right)$ in the case of $\mathrm{B} 0218+357$ and cyanoacetylene $\left(\mathrm{HC}_{3} \mathrm{~N}\right)$ in the case of PKS 1830-211. This may lead to a systematic bias. If we were able to detect $\mathrm{HC}_{3} \mathrm{~N}$ absorption in its ground rotational transition toward PKS 1830-211, for example, the density of the gas traced by this line should only be a few $100 \mathrm{~cm}^{-3}$ and not $2000 \mathrm{~cm}^{-3}$. Otherwise the level populations would be inverted. While $\mathrm{HC}_{3} \mathrm{~N}$ appears to be very weak in $\mathrm{B} 0218+357$, an $\mathrm{H}_{2} \mathrm{CO}$ study like that of Jethava et al. (2007) for B0218+357 is also feasible for PKS 1830-211 to provide a comparison of densities excluding any potential bias caused by the use of different molecular tracers.

In view of our goal to constrain $T_{\mathrm{CMB}}$ (see Sects. 4.1.2 and 4.1.3), it is important that the density derived is not sensitively dependent on the choice of the temperature of the cosmic microwave background. To test this, we also calculated models with $T_{\mathrm{CMB}}=4 \mathrm{~K}$ and $7 \mathrm{~K}$ instead of $5.14 \mathrm{~K}$. Resulting densities are $n\left(\mathrm{H}_{2}\right) \sim 2000 \mathrm{~cm}^{-3}$ and $\sim 1000 \mathrm{~cm}^{-3}$ instead of $\sim 1700 \mathrm{~cm}^{-3}$, when using frequency dependent source covering factors.

\subsubsection{SiO}

$\mathrm{HC}_{3} \mathrm{~N}$ was particularly interesting, because it is a heavy molecule with a small rotational contant. In PKS 1830$211, \mathrm{HC}_{3} \mathrm{~N}$ rotational lines can be observed at multiples of $v \sim 4.8 \mathrm{GHz}$, allowing us to detect several transitions. Small frequencies lead to slow rates of spontaneous decay $\left(A_{i j} \propto v^{3}\right)$ and level populations are already altered by collisional processes in a rather tenuous interstellar medium with $n\left(\mathrm{H}_{2}\right) \sim 1000 \mathrm{~cm}^{-3}$.

There exist a large number of lighter interstellar molecules with higher rotational constants and low apparent optical depths, indicating as in the case of $\mathrm{HC}_{3} \mathrm{~N}$ optically thin absorption. $\mathrm{SiO}$ is one of these species, which exemplifies some effects we have to account for in a thorough analysis of PKS 1830-211. SiO has a five times larger rotational constant than $\mathrm{HC}_{3} \mathrm{~N}$ and a similar electric dipole moment. With $T_{\mathrm{CMB}}=5.14 \mathrm{~K}$ and a density of $2000 \mathrm{~cm}^{-3}$ (Sect. 4.1 and Table 4), LVG modeling (collision rates from Turner et al. 1992) yield in the optically thin limit excitation temperatures of $5.51 \mathrm{~K}$ and $5.21 \mathrm{~K}$ for the $\mathrm{SiO}$ $J=1 \leftarrow 0$ and $2 \leftarrow 1$ lines, respectively. This implies that the densities derived in Sect. 4.1.1 are not drastically modifying line intensities and that, if $\mathrm{SiO}$ arises from the same gas as $\mathrm{HC}_{3} \mathrm{~N}$, the bulk of the excitation is provided by the CMB.
Line-to-continuum ratios of 0.13 or 0.38 are observed in the CS $1 \leftarrow 0$ (Fig. 1) or $\mathrm{HCN}$ and $\mathrm{HCO}^{+} J=2 \leftarrow 1$ lines (Muller \& Guélin 2008), providing firm boundary conditions for the frequency range from the $\mathrm{SiO} 1 \leftarrow 0$ to the $2 \leftarrow 1$ transition. Since the $\mathrm{SiO}$ lines are much weaker, they must be optically thin. With integrated apparent optical depths of $(0.412 \pm 0.021) \mathrm{km} \mathrm{s}^{-1}$ and $(1.44 \pm 0.27) \mathrm{km} \mathrm{s}^{-1}$ for the $1 \leftarrow 0$ and $2 \leftarrow 1$ lines, respectively, we obtain $\tau(2 \leftarrow 1) / \tau(1 \leftarrow 0)=3.50 \pm 0.68$ (Table 5). Assuming Local Thermodynamical Equilibrium (LTE), this results in an excitation temperature of $23 \mathrm{~K}$, with the expected $5 \mathrm{~K}$ (requiring a ratio of 2.2) at a $2 \sigma$ level. We conclude that the noise in the $\mathrm{SiO} J=2 \leftarrow 1$ line is still too high for a definite result. More sensitive $J=2 \leftarrow 1$ data are therefore required.

Using $\mathrm{SiO}$ as an example, we should also note that there exists the possibility that the absorption exclusively arises from a much denser gas component than $\mathrm{HC}_{3} \mathrm{~N}$. Unlike emission lines, the strength of absorption lines does not depend on "critical densities", where timescales of radiative de-excitation match those for collisional excitation. Therefore, a low excitation level caused by large frequencies and dipole moments does not necessarily lead to non-detections and a high ratio of the optical depths between the $2 \leftarrow 1$ and $1 \leftarrow 0$ lines of $\mathrm{SiO}$ at $T_{\mathrm{CMB}} \sim 5.14 \mathrm{~K}$ could be the consequence of chemical differentiation. A test with a more recently measured $\mathrm{SiO} J=4-$ 3 line $\left(\int \tau \mathrm{d} v=0.70 \pm 0.07\right.$; S. Muller, priv. comm.) yields $T_{\text {rot }}=(6.8 \pm 0.3) \mathrm{K}$. While this may indicate that there is no dense $\mathrm{SiO}$ component, we emphasize that half a decade lies between this recent measurement from August/September 2006 and the other data presented here. Variability of the source might thus lead to a larger uncertainty than the given formal error.

Tests with our LVG model show another effect. The excitation of the $J=1 \leftarrow 0$ line rises with increasing density, becoming inverted at $n\left(\mathrm{H}_{2}\right) \sim 6 \times 10^{4} \mathrm{~cm}^{-3}$. While rising, the $J=1 \leftarrow 0$ optical depth decreases, keeping the product $\tau T_{\mathrm{ex}}$ approximately constant. Thus $\tau(2 \leftarrow 1) / \tau(1 \leftarrow 0)$ ratios in excess of four (the maximum for LTE conditions) are possible and may become a sensitive probe of densities related to molecular constituents, unaffected by critical densities. For $T_{\text {kin }}=80 \mathrm{~K}$ and $T_{\mathrm{CMB}}=5.14 \mathrm{~K}$, a density of $1.3 \times 10^{4} \mathrm{~cm}^{-3}$ would reproduce the measured but uncertain ratio of $\sim 3.5$. This density is almost an order of magnitude higher than that derived from $\mathrm{HC}_{3} \mathrm{~N}$.

\subsubsection{Other molecular species}

Here we will analyze opacity ratios between optically thin $J=$ $1 \leftarrow 0$ lines measured by us (Fig. 3) and the corresponding $J=2 \leftarrow 1$ or $J=4 \leftarrow 3$ lines previously reported by Muller et al. (2006, their Table 4). CS has a rotational constant similar to that of $\mathrm{SiO} . \mathrm{HCN}, \mathrm{HCO}^{+}$, and $\mathrm{HNC}$ have rotational constants about twice as large and an order of magnitude larger than that of $\mathrm{HC}_{3} \mathrm{~N}$. This drastically reduces effects caused by collisional excitation as long as these species arise from the same volume as $\mathrm{HC}_{3} \mathrm{~N}$. Columns 5 and 6 of Table 5 show this effect. With the boundary conditions of $T_{\text {kin }}=80 \mathrm{~K}, T_{\mathrm{CMB}}=5.14 \mathrm{~K}$, and $n\left(\mathrm{H}_{2}\right)=2000 \mathrm{~cm}^{-3}$ (Sect. 4.1.1), excitation temperatures for $\mathrm{HCN}, \mathrm{HCO}^{+}$, and $\mathrm{HNC}$ are only slightly larger than $T_{\mathrm{CMB}}$, while for CS the difference is a little larger. The values were obtained from LVG calculations with collision rates of Turner et al. (1992), Flower (1999), and Schöier et al. (2005).

While the signal-to-noise ratio of our $\mathrm{HN}^{13} \mathrm{C} J=1 \leftarrow 0$ line is not high enough to derive a meaningful value, the average of the LTE excitation temperatures of $\mathrm{C}^{34} \mathrm{~S}, \mathrm{H}^{13} \mathrm{CO}^{+}$, and $\mathrm{H}^{13} \mathrm{CN}$ is close to the expected value of $5.14 \mathrm{~K}$. However, deviations of 
Table 5. Integrated optical depths of optically thin lines (see Sects. 4.1.2 and 4.1.3) and resulting excitation temperatures.

\begin{tabular}{|c|c|c|c|c|c|c|}
\hline \multirow[t]{3}{*}{ Species } & \multicolumn{3}{|c|}{$\tau \mathrm{d} V$} & \multicolumn{3}{|c|}{ Excitation temperature } \\
\hline & $J=1 \leftarrow 0$ & $J=2 \leftarrow 1^{a}$ & $J=2 \leftarrow 1 / J=1 \leftarrow 0^{a}$ & $T_{\mathrm{ex}, 1-0}^{b}$ & $T_{\mathrm{ex}, 2-1}^{a, b}$ & $T_{\mathrm{ex}, \mathrm{LTE}}^{c}$ \\
\hline & \multicolumn{2}{|c|}{$\left(\mathrm{km} \mathrm{s}^{-1}\right)$} & & & $(\mathrm{K})$ & \\
\hline $\mathrm{SiO}$ & $0.412 \pm 0.021$ & $1.440 \pm 0.270$ & $3.495 \pm 0.679$ & 5.51 & 5.21 & 23 \\
\hline $\mathrm{C}^{34} \mathrm{~S}^{a}$ & $0.292 \pm 0.020$ & $0.440 \pm 0.040$ & $1.507 \pm 0.172$ & 5.83 & 5.27 & $7.2 \pm 0.4$ \\
\hline $\mathrm{H}^{13} \mathrm{CO}^{+}$ & $2.804 \pm 0.259$ & $2.500 \pm 0.050$ & $0.892 \pm 0.084$ & 5.20 & 5.17 & $3.8 \pm 0.3$ \\
\hline $\mathrm{H}^{13} \mathrm{CN}$ & $1.791 \pm 0.243$ & $2.100 \pm 0.080$ & $1.173 \pm 0.165$ & 5.37 & 5.24 & $4.8 \pm 0.5$ \\
\hline $\mathrm{HN}^{13} \mathrm{C}$ & $0.307 \pm 0.181$ & $0.900 \pm 0.070$ & $2.932 \pm 1.743$ & 5.20 & 5.17 & - \\
\hline $\mathrm{CS}_{-\mathrm{NE}}{ }^{d}$ & $0.080 \pm 0.010$ & $0.280 \pm 0.080$ & $3.500 \pm 1.092$ & - & - & $11 \pm 3$ \\
\hline
\end{tabular}

${ }^{a}$ In the cases of $\mathrm{C}^{34} \mathrm{~S}$ and CS, $J=2 \leftarrow 1$ should be replaced by $J=4 \leftarrow 3$. With the exception of the $\mathrm{SiO} J=2 \leftarrow 1$ line (Fig. 3 ), the $J=2 \leftarrow 1$ and $4 \leftarrow 3$ data were taken from Muller et al. (2006). ${ }^{b} \mathrm{LVG}$ excitation temperatures of optically thin lines with $T_{\text {kin }}=80 \mathrm{~K}, T_{\mathrm{CMB}}=5.14 \mathrm{~K}$, and $n\left(\mathrm{H}_{2}\right)=2000 \mathrm{~cm}^{-3} \cdot{ }^{c}$ Excitation temperatures under conditions of Local Thermodynamical Equilibrium (LTE). For the uncertainty in the estimate from $\mathrm{SiO}$ (this includes so far unpublished $\mathrm{SiO} J=4-3$ data), see Sect. 4.1.2. ${ }^{d} \mathrm{CS}$ absorption toward the north-eastern continuum component, displaced from the main absorption line by $-147 \mathrm{~km} \mathrm{~s}^{-1}$. Since no spatial density has been derived for this feature, Cols. 5 and 6 are left empty.

individual values (see Col. 7 of Table 5) are significant in most cases and are well beyond the estimated uncertainties. Combes \& Wiklind (1999) showed a diagram of previously determined LTE temperatures (their Fig. 4), obtained with the SEST 15-m and IRAM 30-m telescopes. These results also show significant scatter. While LTE temperatures higher then the expected $T_{\mathrm{CMB}}$ value could be explained by additional collisional or radiative excitation (in the case of our $\mathrm{C}^{34} \mathrm{~S}$ results, a density of $n\left(\mathrm{H}_{2}\right)=10^{4} \mathrm{~cm}^{-3}$ would be needed), those ratios below the expected $T_{\mathrm{CMB}}$ value, obtained by Combes \& Wiklind (1999) and by us from linear molecules, are more difficult to interpret. Combes \& Wiklind (1999) suggested that their low values, not including $J=1 \leftarrow 0$ transitions, are caused by the heating of the $1 \leftarrow 0$ and an associated cooling of the $2 \leftarrow 1$ transition. Our data do not confirm this hypothesis, indicating instead that the scatter in the LTE excitation temperatures is much higher than the errors in Col. 7 of Table 5 suggest. A dedicated effort including many more species, among them also asymmetric tops, may solve this problem. So far, the molecular absorption lines toward PKS 1830-211 do not provide the kind of constraints on $T_{\mathrm{CMB}}$, which one is also attempting to obtain in analyzing atomic fine-structure levels in redshifted sources (e.g., Ge et al. 1997; Srianand et al.2000; Molaro et al. 2002).

\subsubsection{The northwestern source}

As already pointed out in Sect. 3, absorption toward the southwestern compact continuum component of PKS 1830-211 is relatively strong. However, a few prominent molecular lines are also detected at a velocity offset of $-147 \mathrm{~km} \mathrm{~s}^{-1}$ toward the northeastern component, which is located behind another spiral arm of the lensing galaxy (e.g., Wiklind \& Combes 1998; Muller et al. 2006). While our $\mathrm{HCO}^{+}$profile (Fig. 1) is not calibrated well enough for an analysis, the CS $J=1 \leftarrow 0$ feature (also Fig. 1) can be compared with the corresponding $J=4 \leftarrow 3$ profile of Muller et al. (2006). The resulting LTE excitation temperature is given in the last line of Table 5. As a caveat, however, we note that the CS spectrum from Muller et al. (2006) was taken in July 1999, more then two years before our measurements (see Muller \& Guélin 2008, for source variability with time). This time interval is longer than for the spectra from the south-western source.

\subsection{The proton-to-electron mass ratio}

\subsubsection{Some general considerations}

As already mentioned in Sect. 1, a comparison of radial velocities from inversion lines of ammonia $\left(\mathrm{NH}_{3}\right)$ with rotational lines of either $\mathrm{NH}_{3}$ or other species can provide sensitive constraints on variations in the proton-to-electron mass ratio $\mu=m_{\mathrm{p}} / m_{\mathrm{e}}$. Following Flambaum \& Kozlov (2007),

$$
\frac{\Delta V}{c}=\frac{z_{\text {inv }}-z_{\text {rot }}}{1+z}=3.46 \times \frac{\Delta \mu}{\mu},
$$

with $z_{\text {inv }}$ and $z_{\text {rot }}$ denoting the apparent redshifts of the inversion and rotational lines, $z$ representing the real redshift of the source, and $\Delta \mu$ providing the deviation relative to the current laboratory value of $\mu$, defined such that a negative value indicates a smaller $\mu$ in the absorbing cloud. In the case where both the $\mathrm{NH}_{3}$ and rotational transitions show absorption in just a single velocity component, defining and measuring the velocity difference $\Delta V$ is straightforward. The more general case where many velocity components exist must be treated differently, by varying all the free parameters, including $\Delta V$, to minimize $\chi^{2}$ in a simultaneous fit to all $\mathrm{NH}_{3}$ and rotational profiles (e.g., Murphy et al. 2008a).

Ammonia and cyanoacetylene $\left(\mathrm{HC}_{3} \mathrm{~N}\right)$ are both molecular high density tracers. Toward PKS 1830-211, the $\mathrm{NH}_{3}$ inversion lines and the rotational $\mathrm{HC}_{3} \mathrm{~N}$ lines are optically thin and observed at similar frequencies. Therefore, in this paper we derive $\Delta \mu / \mu$ by comparing the $\mathrm{NH}_{3}$ and $\mathrm{HC}_{3} \mathrm{~N}$ spectra. Given the fairly low signal-to-noise ratio of these spectra, there is little obvious evidence for more than a single absorbing velocity component across the $\mathrm{NH}_{3}$ and $\mathrm{HC}_{3} \mathrm{~N}$ profiles. We therefore proceed with a simple analysis assuming that only one absorbing velocity component exists, deferring a simultaneous $\chi^{2}$ minimization analysis which incorporates other weak components and other rotational transitions to a later paper. Despite this assumption of a single velocity component, judicious treatment of the uncertainties on its velocity in the $\mathrm{NH}_{3}$ and rotational transitions will ensure our constraint on $\Delta \mu / \mu$ is robust, if not optimally precise.

The $\mathrm{NH}_{3}$ inversion lines were measured with the Green Bank Telescope (GBT), while the $\mathrm{HC}_{3} \mathrm{~N}$ data were obtained at Effelsberg. The Local Standard of Rest velocity scales at both sites are, however, well adjusted (Tifft \& Huchtmeier 1990) and have not been significantly modified in recent years. They refer to a peculiar solar velocity of $20 \mathrm{~km} \mathrm{~s}^{-1}$ toward $\alpha_{1900}=18^{\mathrm{h}}$ and $\delta_{1900}=+30^{\circ}$, corresponding to $V_{\mathrm{LSR}}-V_{\mathrm{HEL}}=12.432 \mathrm{~km} \mathrm{~s}^{-1}$ 
Table 6. Radial velocities (see Sect. 4.2). Uncertainties in the frequencies are small relative to those of the velocities, which were obtained by Gaussian fits ${ }^{a}$.

\begin{tabular}{|c|c|c|c|}
\hline Species & Transition & $\begin{array}{c}\text { Rest } \\
\text { Frequency }(\mathrm{GHz})\end{array}$ & $\begin{array}{c}V_{\mathrm{LSR}} \\
\left(\mathrm{km} \mathrm{s}^{-1}\right)\end{array}$ \\
\hline \multirow[t]{14}{*}{$\mathrm{NH}_{3}$} & $(J, K)=(1,1)$ & 23.694496 & $9.0(0.1)$ \\
\hline & & & $8.6(0.5)$ \\
\hline & $(2,2)$ & 23.722633 & $7.4(0.2)$ \\
\hline & & & $10.5(0.4)$ \\
\hline & $(3,3)$ & 23.870130 & $8.6(0.1)$ \\
\hline & $(4,4)$ & 24.139416 & $8.4(0.2)$ \\
\hline & & & $9.1(0.7)$ \\
\hline & $(5,5)$ & 24.532989 & $8.2(0.3)$ \\
\hline & & & $8.7(0.5)$ \\
\hline & $(6,6)$ & 25.056025 & $8.1(0.2)$ \\
\hline & & & $8.5(0.6)$ \\
\hline & $(7,7)$ & 25.715182 & $10.9(0.4)$ \\
\hline & $(8,9)$ & 27.477943 & $8.8(0.7)$ \\
\hline & $(10,10)$ & 28.604737 & $10.2(2.0)$ \\
\hline \multirow[t]{5}{*}{$\mathrm{HC}_{3} \mathrm{~N}$} & $J=3 \leftarrow 2$ & 27.294289 & $9.0(0.4)$ \\
\hline & $4 \leftarrow 3$ & 36.392324 & $8.2(0.3)$ \\
\hline & $5 \leftarrow 4$ & 45.490314 & $8.0(1.0)$ \\
\hline & $6 \leftarrow 5$ & 54.588247 & $7.7(0.3)$ \\
\hline & $7 \leftarrow 6$ & 63.686052 & $10.0(0.4)$ \\
\hline $\mathrm{SiO}$ & $1 \leftarrow 0$ & 43.423846 & $9.8(0.7)$ \\
\hline $\mathrm{SiO}$ & $2 \leftarrow 1$ & 86.847010 & $8.9(1.8)$ \\
\hline $\mathrm{C}^{34} \mathrm{~S}$ & $1 \leftarrow 0$ & 48.206946 & $9.8(0.6)$ \\
\hline $\mathrm{H}^{13} \mathrm{CO}^{+}$ & $1 \leftarrow 0$ & 86.754288 & $9.7(0.7)$ \\
\hline $\mathrm{H}^{13} \mathrm{CN}$ & $1 \leftarrow 0$ & 86.339922 & $8.8(1.5)$ \\
\hline $\mathrm{HN}^{13} \mathrm{C}$ & $1 \leftarrow 0$ & 87.090873 & $7.4(1.3)$ \\
\hline
\end{tabular}

${ }^{a}$ Frequencies were taken from the JPL catalog $\left(\mathrm{NH}_{3}\right)$, the Cologne Database for Molecular Spectroscopy $\left(\mathrm{HC}_{3} \mathrm{~N}\right)$, and the line list of Lovas (other species). $\mathrm{NH}_{3}$ transitions with two $V_{\text {LSR }}$ values were measured twice (see Henkel et al. 2008).

in the case of PKS 1830-211. Therefore, the velocity measurements listed in Table 6 can be directly compared to estimate $\Delta \mu / \mu$ and its uncertainty, without further velocity corrections.

\subsubsection{Average velocities of $\mathrm{NH}_{3}$ and $\mathrm{HC}_{3} \mathrm{~N}$}

For the single-Gaussian fits to the $\mathrm{NH}_{3}$ transitions, Table 6 reveals somewhat inconsistent centroid velocities. They scatter around the weighted mean velocity over all transitions of $8.65 \mathrm{~km} \mathrm{~s}^{-1}$ with a $\chi^{2}$ value of 117 which, for just 14 degrees of freedom, is far more than expected based on the individual velocity uncertainties. This may indicate the presence of more than one velocity component. Despite the large range of $\mathrm{NH}_{3}$ excitation states observed by Henkel et al. (2008) - some $1030 \mathrm{~K}$ - we do not observe a trend in velocity with excitation state: For the three lowest $\mathrm{NH}_{3}$ inversion lines, the unweighted mean velocity is $8.82 \pm 0.45 \mathrm{~km} \mathrm{~s}^{-1}$ and for the higher excited ones we find a mean of $8.94 \pm 0.27 \mathrm{~km} \mathrm{~s}^{-1}$ (the errors are standard deviations on the means). Indeed, the larger than expected scatter in fitted velocities for the different $\mathrm{NH}_{3}$ transitions seems to be random in sign and magnitude. A similar additional scatter is also observed in the rotational $\mathrm{HC}_{3} \mathrm{~N}$ transitions (Fig. 2). These cover only a small amount of excitation, with the highest level, $J=7$, being just $\sim 12 \mathrm{~K}$ above the ground state.
Given the velocity scatter in the transitions of $\mathrm{NH}_{3}$ and $\mathrm{HC}_{3} \mathrm{~N}$, constraining the variation of $\mu$ is best done with conservative velocity and uncertainty estimates which naturally incorporate the observed scatter. The simplest such velocity estimator is obviously the unweighted mean velocity and its standard deviation which, for the $\mathrm{NH}_{3}$ transitions in Table 6, are $8.90 \pm$ $0.24 \mathrm{~km} \mathrm{~s}^{-1}$, and for the $\mathrm{HC}_{3} \mathrm{~N}$ transitions, are $8.58 \pm 0.37 \mathrm{~km} \mathrm{~s}^{-1}$. Of course, the statistical velocity uncertainties quoted in Table 6 for some transitions are so high that including them in the mean velocity calculation is likely to decrease the reliability of the mean. We therefore reject transitions with velocity uncertainties larger than the root-mean-square (rms) velocity variation for each species, 0.91 and $0.83 \mathrm{~km} \mathrm{~s}^{-1}$ for $\mathrm{NH}_{3}$ and $\mathrm{HC}_{3} \mathrm{~N}$ respectively. With this criterion, the $\mathrm{NH}_{3}(10,10)$ and $\mathrm{HC}_{3} \mathrm{~N} 5 \leftarrow 4$ transitions are rejected. The final mean velocity for the remaining $\mathrm{NH}_{3}$ transitions is $8.81 \pm 0.23 \mathrm{~km} \mathrm{~s}^{-1}$ and, for the remaining $\mathrm{HC}_{3} \mathrm{~N}$ transitions, is $8.73 \pm 0.43 \mathrm{~km} \mathrm{~s}^{-1}$.

\subsubsection{Resulting limits for a variation of $\mu$}

Using these final clipped mean velocities, $\Delta V=0.08 \pm$ $0.49 \mathrm{~km} \mathrm{~s}^{-1}$. Equation (1) then provides our $1-\sigma$ constraint on the variation in $\mu, \Delta \mu / \mu=(+0.08 \pm 0.47) \times 10^{-6}$. Since the quoted uncertainty derives entirely from the scatter in the individual transition velocities, this should be a reasonably robust error estimate. Nevertheless, given that we have used only single Gaussian fits to the absorption profiles and that there is some scatter in the individual transition velocities, we quote our final result as a 3- $\sigma$ upper limit on variation in $\mu$ at the absorption redshift of $z=0.89$,

$|\Delta \mu / \mu|<1.4 \times 10^{-6}$.

For comparison with laboratory constraints on variations in $\mu$, and in the absence of a reliable model for how $\mu$ might be expected to vary with cosmological time, it is common, if not well motivated, to assume that any variation is linear in time. Hence, our upper limit on variation in $\mu$ translates to a $3-\sigma$ upper limit on its time variation

$|\dot{\mu} / \mu|<2.0 \times 10^{-16} \mathrm{yr}^{-1}$

over the past $7.0 \mathrm{Gyr}$.

There is yet another study on the proton-to-electron mass ratio in the main lens of PKS 1830-211. From a comparison of the ammonia inversion lines (Henkel et al. 2008) with its $(J$, $K)=(1,0) \leftarrow(0,0)$ rotational transition, Menten et al. (2008) find consistency, at a $1 \sigma$ level, of $\Delta \mu / \mu=1.9 \times 10^{-6}$. The strength of this study is its focus on lines with different dependencies on $\mu$ arising from the same molecular species. On the other hand, the ratio between the frequencies of the rotational and inversion lines is $\sim 25$, thus leading to potentially significant differences in the morphology and the covering factor of the background radio and submillimeter continuum. While such systematic differences cannot be quantified on the basis of a single rotational line, the given uncertainty of the resulting $\Delta \mu / \mu$ value is dominated by the limited signal-to-noise ratio of the rotational line.

\subsubsection{Potential caveats}

The consistency of $\mathrm{NH}_{3}$ and $\mathrm{HC}_{3} \mathrm{~N}$ radial velocities is remarkable in view of a number of effects, which might exert a significant influence on our results. Having carefully avoided the use of optically thick transitions, these are (1) time variability of the continuum source, (2) a frequency dependent continuum 
morphology, (3) hyperfine structure, (4) chemistry, and (5) inhomogeneities in temperature and density inside a cloud of size $\gtrsim 10$ pc (Carilli et al. 1998).

(1) A time variable continuum source may lead to different linesof-sight and thus to different radial velocities at different epochs. Not much of this was seen in spite of the existence of monitoring programs (Wiklind \& Combes 1998; Muller et al.2006; Muller \& Guélin 2008) or repeated measurements of ammonia lines (Table 6 and Henkel et al. 2008).

(2) $\mathrm{The} \mathrm{NH}_{3}$ and $\mathrm{HC}_{3} \mathrm{~N}$ transitions used in this study are much closer in frequency than those chosen by Murphy et al. (2008a) and Menten et al. (2008). Also, in PKS 1830-211, the source covering factor may be less frequency dependent than in $\mathrm{B} 0218+357$ (Sect. 4.1.1). Nevertheless, $\mathrm{NH}_{3}$ and $\mathrm{HC}_{3} \mathrm{~N}$ frequencies differ by factors of 1.0-2.5. While no direct effect is apparent, it remains a source of uncertainty which cannot be quantified.

(3) In particular $\mathrm{NH}_{3}$ velocities would be greatly affected by hyperfine (hf) splitting, if strong non-LTE effects would occur. A main group of hf-components is surrounded by four satellite groups, displaced by about \pm 10 and $\pm 20 \mathrm{~km} \mathrm{~s}^{-1}$. The higher the energy above the ground state of an inversion line, the weaker the satellite features relative to the main group (e.g., the satellites account for $\sim 2.5 \%$ of the total absorption in the $(J, K)=(7,7)$ line $)$. The above made comparison of average velocities of the three lowest with the seven higher excited $\mathrm{NH}_{3}$ inversion lines does not show a significant shift in velocity. Deviations from LTE intensity ratios of the various components are only expected in the case of a significant population of the non-metastable inversion states $(J>K)$, which requires extremely high densities or intense radiation fields (Stutzki et al. 1984; Stutzki \& Winnewisser 1985a,b). Non-LTE effects leading to $\mathrm{HC}_{3} \mathrm{~N}$ velocity shifts as a function of rotational quantum number $J$ are also not obvious. In this case, the $J=3 \leftarrow 2$ line would be the most critical, while the hf-structure of the $7 \leftarrow 6$ line is far too compact to yield any significant shifts.

(4) Chemistry is a more difficult issue, because there is no way to estimate its effect on our data. $\mathrm{NH}_{3}$ and $\mathrm{HC}_{3} \mathrm{~N}$ may have different spatial distributions, which is most impressively exemplified by the nearby prototypical dark cloud TMC-1, where peaks of line emission are offset by 7 arcmin (e.g., Olano et al. 1988). $\mathrm{HC}_{3} \mathrm{~N}$ is a molecule representing a young chemical age, while $\mathrm{NH}_{3}$ refers to a chemically more evolved stage of cloud chemistry (e.g., Dickens et al. 2001; Hirota et al. 2002).

(5) Since $\mathrm{NH}_{3}$ is covering an enormous range of excitation without showing any significant change in radial velocity, changes in temperature and density may also not play an important role.

\subsubsection{Summary and outlook}

To summarize, we either have the unlikely scenario in which different effects cancel each other out, or all of these effects are minor. In order to broaden the scope of our study and to avoid relying entirely on the rotational transitions of $\mathrm{HC}_{3} \mathrm{~N}$, we may also use the $J=1 \leftarrow 0$ and $2 \leftarrow 1$ lines presented in Fig. 3. We should note, however, that for these lines, frequencies are significantly higher than for $\mathrm{NH}_{3}$ and $\mathrm{HC}_{3} \mathrm{~N}$. The unweighted mean rotational velocity then becomes $9.07 \pm 0.38 \mathrm{~km} \mathrm{~s}^{-1}$. The velocity difference amounts to $\Delta V=+0.26 \pm 0.44 \mathrm{~km} \mathrm{~s}^{-1}$ relative to $\mathrm{NH}_{3}$, while for $\mathrm{HC}_{3} \mathrm{~N}$ we have found $-0.08 \pm 0.49 \mathrm{~km} \mathrm{~s}^{-1}$. These differences highlight potential systematic effects, caused by a time variable continuum background, by a frequency dependent continuum morphology, by non-LTE effects in lines with hyperfine structure, by astrochemistry, and by the specific excitation requirements of individual lines. The large number of analyzed molecular transitions, covering a wide parameter space, ensures that systematic discrepancies in velocity are within $1 \mathrm{~km} \mathrm{~s}^{-1}$, corresponding to $\Delta \mu / \mu<10^{-6}$.

Our constraint on variations of the proton-to-electron mass ratio $\mu$ differs in several ways from that given by Murphy et al. (2008a) for B0218+357 at $z \sim 0.68$ (this study was also based on a comparison of ammonia $\left(\mathrm{NH}_{3}\right)$ inversion lines with rotational transitions of other molecular species). (1) The frequencies of the lines measured by us are much more similar than in the previous study, minimizing effects caused by a frequency dependent continuum background morphology on the observed column. (2) All our analyzed lines are optically thin, avoiding modified lineshapes due to saturation and (3) our number of observed transitions is much larger. This may result in an evaluation of hidden systematic errors, which is more realistic than any estimate from multi-velocity component fits of a few spectral lines only. (4) The time interval between our measurements of inversion and rotational lines is smaller, possibly reducing systematic effects caused by a time variable radio continuum background. Note, however, that this may be compensated by a higher variability of our source.

There are also two weaknesses with respect to the study of Murphy et al. (2008a). (1) The excitation of most inversion lines analyzed by us is much higher than that of the rotational lines. While no trend of radial velocities as a function of excitation is seen by us, the study of Murphy et al. (2008a) is confined to inversion lines of modest excitation and is definitely less sensitive to potential discrepancies with respect to excitation. (2) We did not yet provide a multi-component spectral analysis of the entire dataset, accounting for velocity structure and hyperfine splitting. This is the missing step toward a complete analysis of the spectra and will be discussed in a forthcoming paper, focusing entirely on potential variations of $\mu$.

\section{Conclusions}

Our observations of molecular lines at redshift $z \sim 0.89$ toward the gravitational lens system PKS 1830-211 reveal the following main results:

- Cyanoacetylene $\left(\mathrm{HC}_{3} \mathrm{~N}\right)$ is detected in absorption in five rotational lines. The $J=7 \leftarrow 6$ line may have been detected for the first time in the interstellar medium. $\mathrm{CS}, \mathrm{C}^{34} \mathrm{~S}, \mathrm{HCO}^{+}$, $\mathrm{HC}^{13} \mathrm{O}^{+}, \mathrm{H}^{13} \mathrm{CN}$, and $\mathrm{SiO}$ absorption are also detected, in their ground rotational transitions.

- A Large Velocity Gradient analysis of the $\mathrm{HC}_{3} \mathrm{~N}$ spectra yields, adopting $T_{\text {kin }}=80 \mathrm{~K}$ from ammonia $\left(\mathrm{NH}_{3}\right)$, a density of $n\left(\mathrm{H}_{2}\right) \sim 2600 \mathrm{~cm}^{-3}$ for a background source covering factor $f_{\mathrm{c}}$, which is independent of frequency. $\mathrm{CS}, \mathrm{HCO}^{+}$, and $\mathrm{OH}$ data constrain its frequency dependence to $f_{\mathrm{c}} \propto v^{0.0 \ldots . .5}$. This holds for the frequency range between $26 \mathrm{GHz}$ and $94 \mathrm{GHz}$. For a maximal $f_{\mathrm{c}}$ exponent of $0.5, n\left(\mathrm{H}_{2}\right)$ is reduced to $\sim 1700 \mathrm{~cm}^{-3}$.

- The gas density determined using $\mathrm{HC}_{3} \mathrm{~N}$ is low enough that molecules with higher rotational constants should be mainly excited by the cosmic microwave background. Therefore, we compare our $\mathrm{C}^{34} \mathrm{~S}, \mathrm{H}^{13} \mathrm{CO}^{+}$, and $\mathrm{H}^{13} \mathrm{CN} J=1 \leftarrow 0$ data with the corresponding $J=2 \leftarrow 1$ lines $(J=4 \leftarrow 3$ in the 
case of $C^{34} S$ ) presented by Muller et al. (2006). While the average excitation temperature is consistent with the expected temperature of the microwave backgrund, $T_{\mathrm{CMB}}=5.14 \mathrm{~K}$, deviations of individual values (3-7 K) far exceed expected uncertainties.

- For the weak north-eastern absorption component, a comparison of the CS $J=1 \leftarrow 0$ and $4 \leftarrow 3$ transitions yields an excitation temperature of $(11 \pm 3) \mathrm{K}$. The comparison is, however, based on data, which were taken at epochs more than two years apart.

- From a comparison of the radial velocities of five rotational transitions of $\mathrm{HC}_{3} \mathrm{~N}$ with ten inversion transitions of ammonia, the proton-to-electron mass ratio $\mu$ of the lens at $z=0.89$ deviates by $|\Delta \mu / \mu|<1.4 \times 10^{-6}$ from the local value with $3-\sigma(99.7 \%)$ confidence. Assuming that any variation in $\mu$ evolves linearly with cosmological time, this corresponds to $|\dot{\mu} / \mu|<2.0 \times 10^{-16} \mathrm{yr}^{-1}$. The large number of analyzed optically thin molecular transitions, covering a wide range of physical and chemical parameters, indicates that systematic effects should be $<1 \mathrm{~km} \mathrm{~s}^{-1}$, corresponding to an uncertainty of $<10^{-6}$ in $\Delta \mu / \mu$.

- Comparing the main lens of PKS 1830-211 with that of B0218+357, the molecular gas toward PKS 1830-211 is warmer and appears to be denser, while its molecular source covering factor may be slightly less frequency dependent at $\mathrm{cm}$ - and mm-wavelengths.

Acknowledgements. It is a pleasure to thank J. N. Chengalur, S. Thorwirth, and C. M. Walmsley for useful discussions and critical reading of the manuscript. M. T. M. thanks the Australian Research Council for a QEII Fellowship (DP0877998). We used NASA's Astrophysical Data System (ADS), the Cologne Database for Molecular Spectroscopy (CDMS; see Müller et al. 2001, 2005), the JPL Catalog (http://spec.jpl.nasa.gov/ftp/pub/catalog/catform. html), and the line lists of Lovas (1992) and Coudert \& Roueff (2006).

\section{References}

Bahcall, J. N., Sargent, W. L. W., \& Schmidt, M. 1967, ApJ, 149, L11 Battistelli, E. S., De Petris, M., Lamagna, L., et al. 2002, ApJ, 580, L101 Calmet, X., \& Fritzsch, H. 2002, Eur. Phys. J. C, 24, 639

Carilli, C. L., Menten, K. M., Reid, M. J., Rupen, M., \& Claussen, M. 1998, in Radio Emission from Galactic and Extragalactiv Compact Sources, ed. A. Zensus et al., ASP Conf. Ser., 144, 317

Carilli, C. L., Menten, K. M., Stocke, J. T., et al. 2000, Phys. Rev. Lett., 85, 5511

Chand, H., Srianand, R., Petitjean, P., \& Aracil, B. 2004, A\&A, 417, 853

Chengalur, J. N., \& Kanekar, N. 2003, Phys. Rev. Lett., 91, 241302

Chengalur, J. N., de Bruyn, A. G., \& Narasimha, D. 1999, A\&A, 343, L79

Combes, F., \& Wiklind, T. 1995, A\&A, 303, L61

Combes, F., \& Wiklind, T. 1999, in Highly Redshifted Radio Lines, ed. C. Carilli et al., ASP Conf. Ser., 156, 210

Courbin, F., Meylan, G., Kneib, J.-P., \& Lidman, C. 2002, ApJ, 575, 95

Cowie, L. L., \& Songaila, A. 1995, ApJ, 453, 596

Coudert, L. H., \& Roueff, E. 2006, A\&A, 449, 855

Dent, T. 2008, Phys. Rev. D, 78, 103518

De Rosa, A., Piro, L., Tramacere, A., et al. 2005, A\&A, 438, 121

Dickens, J. E., Langer, W. D., \& Velusamy, T. 2001, ApJ, 558, 693

Drinkwater, M. J., Webb, J. K., Barrow, J. D., \& Flambaum, V. V. 1998, MNRAS, 295,457

Fixsen, D. J., \& Mather, J. C. 2002, ApJ, 581, 817

Flambaum, V. V. 2008, Eur. Phys. J. Special Topics, 163, 159

Flambaum, V. V., \& Kozlov, M. G. 2007, Phys. Rev. Lett., 98, 240801

Flambaum, V. V., Leinweber, D. B., Thomas, A. W., \& Young, R. D. 2004, Phys. Rev., 69, 115006

Flower, D. R. 1999, MNRAS, 305, 651

Frye, B., Welch, W. J., \& Broadhurst, T. 1997, ApJ, 478, L25

García-Berro, E., Isern, J., \& Kubyshin, Y. A. 2007, A\&AR, 14, 113

Ge, J., Bechtold, J., \& Black, J. H. 1997, ApJ, 474, 67

Gerin, M., Phillips, T. G., Benford, D. J., et al. 1997, ApJ, 488, L31

Green, S., \& Chapman, S. 1978, ApJS, 37, 169
Henkel, C., Jethava, N., Kraus, A., et al. 2005, A\&A, 440, 893

Henkel, C., Braatz, J. A., Menten, K. M., \& Ott, J. 2008, A\&A, 485, 451

Hirota, T., Ito, T., \& Yamamoto, S. 2002, ApJ, 565, 359

Impellizzeri, C. M. V., McKean, J. P., Castangia, P., et al. 2008, Nature, 456, 927

Ivanchik, A., Petitjean, P., Varshalovich, D., et al. 2005, A\&A, 440, 45

Jauncey, D. L., Reynolds, J. E., Tzioumis, A. K., et al. 1991, Nature, 352, 132

Jethava, N., Henkel, C., Menten, K. M., Carilli, C. L., \& Reid, M. J. 2007, A\&A, 472,435

Jin, C., Garrett, M. A., Nair, S., et al. 2003, MNRAS, 340, 1309

Kanekar, N., Chengalur, J. N., \& Ghosh, T. 2004, Phys. Rev. Lett., 93, 051302

Kanekar, N., Carilli, C. L., Langston, G. I., et al. 2005, Phys. Rev. Lett., 95, 261301

King, J. A., Webb, J. K., Murphy, M. T., \& Carswell, R. F. 2008, Phys. Rev. Lett., 101,251304

Koopmans, L. V. E., \& de Bruyn, A. G. 2005, MNRAS, 360, L6

Langacker, P., Segré, G., \& Strassler, J. 2002, Phys. Lett. B, 528, 121

Levshakov, S. A., Dessauges-Zavadsky, M., D’Odorico, S., \& Molaro, P. 2002, MNRAS, 333, 373

Levshakov, S. A., Centurión, M., Molaro, P., et al. 2006, A\&A, 449, 879

Levshakov, S. A., Molaro, P., Lopez, S., et al. 2007, A\&A, 466, 1077

Lidman, C., Courbin, F., Meylan, G., et al. 1999, ApJ, 514, L57

Lima, J. A. S., Silva, A. I., \& Viegas, S. M. 2000, MNRAS, 312, 747

Lobanov, A. P. 1998, A\&A, 330, 79

Lovas, F. J. 1992, J. Phys. Chem. Ref. Data, 21, 181

Mauersberger, R., Henkel, C., \& Sage, L. J. 1990, A\&A, 236, 63

Menten, K. M., Carilli, C., \& Reid, M. J. 1999, in Highly Redshifted Radio Lines, ed. C. Carilli, et al., ASP Conf. Ser., 156, 218

Menten, K. M., Güsten, R., Leurini, S., et al. 2008, A\&A, 492, 725

Molaro, P., Levshakov, S. A., Dessauges-Zavadsky, M., \& D’Odorico, S. 2002, A\&A, 381, L64

Müller, H. S. P., Thorwirth, S., Roth, D. A., \& Winnewisser, G. 2001, A\&A, 370, L49

Müller, H. S. P., Schlöder, F., Stutzki, J., \& Winnewisser, G. 2005, J. Mol. Struct., 742,215

Muller, S., \& Guélin, M. 2008, A\&A, 491, 739

Muller, S., Guélin, M., Dumke, M., Lucas, R., \& Combes, F. 2006, A\&A, 458, 417

Murphy, M. T., Webb, J. K., Flambaum, V. V., et al. 2001, MNRAS, 327, 1244 Murphy, M. T., Webb, J. K., \& Flambaum, V. V. 2003, MNRAS, 345, 609

Murphy, M. T., Flambaum, V. V., Muller, S., \& Henkel, C. 2008a, Sci, 320, 1611

Murphy, M.T., Webb, J. K., \& Flambaum, V. V. 2008b, MNRAS, 384, 1053

Nair, S., Narasimha, D., \& Rao, A. P. 1993, ApJ, 407, 46

Olano, C. A., Walmsley, C. M., \& Wilson, T. L. 1988, A\&A, 196, 194

Rosenband, T., Hume, D. B., Schmidt, P. O., et al. 2008, Science, 319, 1808

Reinhold, E., Buning, R., Hollenstein, U., et al. 2006, Phys. Rev. Lett., 96, 151101

Schöier, F. L., van der Tak, F. F. S., van Dishoeck, E. F., \& Black, J. H. 2005, A\&A, 432, 369

Spergel, D. N., Verde, L., Peiris, H. V., et al. 2003, ApJS, 148, 175

Spergel, D. N., Bean, R., \& Doré, O., et al. 2007, ApJS, 170, 377

Srianand, R., Petitjean, P., \& Ledoux, C. 2000, Nature, 408, 931

Stutzki, J., \& Winnewisser, G. 1985a, A\&A, 144, 1

Stutzki, J., \& Winnewisser, G. 1985b, A\&A, 144, 13

Stutzki, J., Olberg, M., Winnewisser, G., Jackson, J. M., \& Barrett, A. H. 1984, A\&A, 139, 258

Swift, J. J., Welch, W. J., \& Frye, B. L. 2001, ApJ, 549, L29

Tifft, W. G., \& Huchtmeier, W. K. 1990, A\&AS, 84, 47

Turner, B. E., Chan, K.-W., Green, S., \& Lubowich, D. A. 1992, ApJ, 399, 114

Tzanavaris, P., Webb, J. K., Murphy, M. T., Flambaum, V. V., \& Curran, S. J. 2005, Phys. Rev. Lett., 95, 041301

Tzanavaris, P., Murphy, M. T., Webb, J. K., Flambaum, V. V., \& Curran, S. J. 2007, MNRAS, 374, 634

Uzan, J. P. 2003, Rev. Mod. Phys., 75, 403

Van Ommen, T. D., Jones, D. L., Preston, R. A., \& Jauncey, D. L. 1995, ApJ, 444,561

Varshalovich, D. A., \& Levshakov, S. A, 1993, J. Exp. Theor. Phys. Lett., 58, 237

Webb, J. K., Flambaum, V. V., Churchill, C. W., Drinkwater, M. J., \& Barrow, J. D. 1999, Phys. Rev. Lett., 82, 884

Webb, J. K., Murphy, M. T., Flambaum, V. V., et al. 2001, Phys. Rev. Lett., 87, 091301

Wiklind, T., \& Combes, F. 1994, A\&A, 286, L9

Wiklind, T., \& Combes, F. 1995, A\&A, 299, 382

Wiklind, T., \& Combes, F. 1996a, A\&A, 315, 86

Wiklind, T., \& Combes, F. 1996b, Nature, 379, 139

Wiklind, T., \& Combes, F. 1998, ApJ, 500, 129

Winn, J. N., Kochanek, C. S., McLeod, B. A., et al. 2002, ApJ, 575, 103 